\section{Environmental Contamination from E-cigarette, Cigarette, Cigar, and Cannabis Products at 12 High Schools - San Francisco Bay Area, 2018-2019}

\author{
Jeremiah Mock, $\mathrm{PhD}^{1,2}$; Yogi H. Hendlin, $\mathrm{PhD}^{3,4}$
}

The United States is experiencing an epidemic of lung injury associated with youth electronic cigarette (e-cigarette) use, or vaping (1); in 2018, 20.8\% of U.S. high school students reported currently using e-cigarettes $(1)$. E-cigarette products such as Juul, a popular device that delivers nicotine and flavors, ${ }^{*}$ are used by students at schools, including in classrooms and bathrooms. ${ }^{\dagger}$ Use of flavored e-cigarettes by youths has become an increasing concern (2). A recent analysis of the National Youth Tobacco Survey showed that among high school students who currently used e-cigarettes, the percentage who used flavored e-cigarettes increased from $65.1 \%$ in 2014 to $67.8 \%$ in 2018 (3). In $2018,8.1 \%$ of high school students currently smoked cigarettes, and $45.7 \%$ of those students smoked menthol cigarettes. In addition, $7.6 \%$ of high school students currently smoked cigarillos, little cigars, or cigars, $43.6 \%$ of whom used flavored varieties of these products $(1,3)$. Many youths also use cigars to make marijuana blunts (i.e., cigarillos with the tobacco removed and replaced with marijuana) (4), and some use manufactured disposable cannabis products (e.g., vape pens, vaporizer cartridges, oils, and concentrates) (5). Waste from e-cigarette products can contain plastics, nicotine, heavy metals, other chemical toxins, and hazardous lithium-ion batteries $(6,7)$. The toxicity of combustible tobacco product waste from cigarettes (e.g., plastic cellulose acetate, nicotine, formaldehyde, lead, and cadmium) is well established (8). Cannabis product waste can include plastics, metals, electronic components, and batteries.

A garbology ${ }^{\S}$ study of environmental contamination from e-cigarette product waste, combustible tobacco product waste, and cannabis product waste was conducted using a purposively selected, nonrandom sample of 12 public high schools with a total enrollment of 18,831 students in Alameda, Contra Costa, Marin, and San Francisco counties in California. Using 2016 data from the National Center for Education Statistics, researchers stratified schools by the percentages of students

\footnotetext{
${ }^{*}$ Menthol is one of the types of tobacco-product flavoring. https://www. tobaccofreekids.org/assets/factsheets/0394.pdf.

${ }^{\dagger}$ https://truthinitiative.org/research-resources/emerging-tobacco-products/ nearly-1-5-youth-say-they-have-seen-juul-used-school.

$\$$ The ethno-archeological study of a community or cultural group by analyzing its waste.
}

from low-income families (i.e., those with students eligible for free or reduced-price lunch). At each school, researchers systematically scanned the student parking lots and exterior school perimeter areas once during July 2018-April 2019 to collect all e-cigarette product waste, combustible tobacco product waste, and cannabis product waste found on the ground.

Overall, 893 waste items were collected, including 172 (19\%) e-cigarette product waste items (nearly all were Juul or Juul-compatible pods and pod caps) (Table). Almost all Juul or Juul-compatible pods and caps were found at schools with predominantly middle- and upper-income student populations. Among 74 (43\%) Juul or Juul-compatible color-coded flavor caps, 73 (99\%) were from flavored pods other than tobacco flavor. Overall, 47 (64\%) pod caps were from mintflavored (e.g., Cool Mint) and other menthol-flavored (e.g., Cool Cucumber and Classic Menthol) pods. Additional scans were conducted at one upper-income area school beginning 3 months after Juul Laboratories announced it was removing flavors (except Cool Mint and Classic Menthol) from retail distribution. These additional scans yielded 127 mint, 20 mango, four fruit Juul or Juul-compatible pod caps, and three yellow (banana or mango) Juul-compatible caps.

At four high schools with populations composed predominantly of lower-income African-American and Latino students, eight e-cigarette product waste items were collected, in addition to 71 little cigar or cigarillo plastic wrappers and mouthpieces, $94 \%$ of which were from flavored products. No little cigar or cigarillo items were found at schools in upperincome communities.

Across all schools, 620 cigarette butts were collected, including 403 (65\%) from recently smoked cigarettes that were identifiable. Among these, 168 (42\%) were menthol. At low-, middle-, and upper-income schools, identifiable menthol butts accounted for $60 \%, 38 \%$, and $28 \%$, respectively, of all identifiable cigarette butts. Fourteen cannabis product waste items were found, including vaporizer pens, cartridges, and packaging from high-potency pineapple- and lemon-flavored cannabis oil concentrate vaporizer cartridges.

E-cigarette waste and combustible tobacco product waste contaminate the Bay Area high schools studied and confirm use of these products by high school students. Cannabis product waste represents an emerging issue. The large proportions of flavored products identified in this study are consistent with findings from other studies showing high prevalence rates of flavored e-cigarette and combustible tobacco product use

Sttps://nces.ed.gov/ccd/schoolsearch/. 
TABLE. Electronic cigarette, combustible tobacco product, and cannabis product waste collected at 12 high schools, by percentage of students from low-income families ${ }^{*},+$ and other demographic characteristics — San Francisco Bay Area, 2018-2019

\begin{tabular}{|c|c|c|c|c|c|c|c|c|c|c|c|c|c|c|c|c|}
\hline \multirow{2}{*}{$\begin{array}{l}\text { Characteristic } \\
\text { Public high school no. }\end{array}$} & \multicolumn{5}{|c|}{ Low income* } & \multicolumn{5}{|c|}{ Middle income* } & \multicolumn{5}{|c|}{ Upper income* } & \multirow{2}{*}{$\begin{array}{l}\text { Total } \\
1-12\end{array}$} \\
\hline & 1 & 2 & 3 & 4 & $\begin{array}{l}\text { Subtotals and } \\
\text { averages for } \\
\text { schools } 1-4\end{array}$ & 5 & 6 & 7 & 8 & $\begin{array}{l}\text { Subtotals and } \\
\text { averages for } \\
\text { schools 5-8 }\end{array}$ & 9 & 10 & 11 & 12 & $\begin{array}{l}\text { Subtotals and } \\
\text { averages for } \\
\text { schools } 9-12\end{array}$ & \\
\hline Student population ${ }^{* \dagger}+$ & 1,528 & 1,583 & 865 & 1,210 & 5,186 & 2,685 & 1,117 & 1,296 & 3,205 & 8,303 & 1,076 & 1,077 & 1,419 & 1,770 & 5,342 & 18,831 \\
\hline $\begin{array}{l}\text { Students from low-income } \\
\text { families (\%) }\end{array}$ & 92 & 88 & 81 & 56 & 79.3 & 40 & 32 & 32 & 28 & 33.0 & 20 & 7 & 9 & 4 & 10.0 & 40.8 \\
\hline $\begin{array}{l}\text { Students learning English as a } \\
\text { second language (\%) }\end{array}$ & 43 & 22 & 37 & 22 & 31.0 & 2 & 11 & 11 & 6 & 7.5 & 7 & 1 & 2 & 1 & 2.8 & 13.8 \\
\hline Female students (\%) & 51 & 47 & 42 & 50 & 47.5 & 59 & 46 & 53 & 51 & 52.3 & 46 & 50 & 49 & 49 & 48.5 & 49.4 \\
\hline \multicolumn{17}{|c|}{ Percentage of students, by race/ethnicity ${ }^{\dagger, \S}$} \\
\hline Latino or Hispanic & 86 & 27 & 64 & 64 & 60.3 & 10 & 40 & 36 & 21 & 26.8 & 26 & 11 & 9 & 11 & 14.3 & 33.8 \\
\hline African American or black & 5 & 35 & 25 & 1 & 16.5 & 2 & 2 & 3 & 19 & 6.5 & 3 & 2 & 5 & 1 & 2.8 & 8.6 \\
\hline White & 1 & 2 & 2 & 29 & 8.5 & 15 & 45 & 50 & 40 & 37.5 & 60 & 77 & 69 & 74 & 70.0 & 38.7 \\
\hline Asian & 6 & 33 & 7 & 5 & 12.8 & 65 & 8 & 5 & 9 & 21.8 & 5 & 3 & 9 & 7 & 6.0 & 13.5 \\
\hline American Indian or Alaska Native & $<1$ & $<1$ & $<1$ & $<1$ & $<1$ & $<1$ & 1 & 1 & $<1$ & $<1$ & 1 & $<1$ & $<1$ & $<1$ & $<1$ & $<1$ \\
\hline $\begin{array}{l}\text { Hawaiian Native or Pacific } \\
\text { Islander }\end{array}$ & $<1$ & 1 & 1 & $<1$ & $<1$ & 1 & $<1$ & $<1$ & $<1$ & $<1$ & $<1$ & $<1$ & $<1$ & $<1$ & $<1$ & $<1$ \\
\hline Multiracial & $<1$ & 2 & 1 & $<1$ & 1.0 & 7 & 2 & 5 & 11 & 6.3 & 4 & 6 & 8 & 7 & 6.3 & 4.3 \\
\hline Total no. of waste items & 18 & 71 & 232 & 38 & 359 & 67 & 39 & 12 & 30 & 148 & 15 & 33 & 118 & 220 & 386 & 893 \\
\hline $\begin{array}{l}\text { Total no. of Juul and } \\
\text { Juul-compatible items }\end{array}$ & 0 & 0 & 2 & 6 & 8 & 0 & 6 & 3 & 3 & 12 & 3 & 6 & 15 & 128 & 152 & 172 \\
\hline Juul or Juul-compatible pods & 0 & 0 & 1 & 3 & 4 & 0 & 1 & 0 & 1 & 2 & 0 & 1 & 7 & 33 & 41 & 47 \\
\hline $\begin{array}{l}\text { Juul or Juul-compatible pod } \\
\text { black end-caps }\end{array}$ & 0 & 0 & 1 & 3 & 4 & 0 & 0 & 0 & 0 & 0 & 1 & 1 & 4 & 39 & 45 & 49 \\
\hline $\begin{array}{l}\text { Juul or Juul-compatible Classic } \\
\text { Tobacco cap }\end{array}$ & 0 & 0 & 0 & 0 & 0 & 0 & 1 & 0 & 0 & 1 & 0 & 0 & 0 & 0 & 0 & 1 \\
\hline $\begin{array}{l}\text { Juul or Juul-compatible Virginia } \\
\text { Tobacco cap }\end{array}$ & 0 & 0 & 0 & 0 & 0 & 0 & 0 & 0 & 0 & 0 & 0 & 0 & 0 & 1 & 1 & 1 \\
\hline $\begin{array}{l}\text { Juul or Juul-compatible Cool } \\
\text { Mint cap }\end{array}$ & 0 & 0 & 0 & 0 & 0 & 0 & 0 & 0 & 2 & 2 & 2 & 4 & 0 & 31 & 37 & 39 \\
\hline $\begin{array}{l}\text { Juul or Juul-compatible Mango } \\
\text { cap }\end{array}$ & 0 & 0 & 0 & 0 & 0 & 0 & 4 & 1 & 0 & 5 & 0 & 0 & 4 & 10 & 14 & 19 \\
\hline $\begin{array}{l}\text { Juul or Juul-compatible Cool } \\
\text { Cucumber cap }\end{array}$ & 0 & 0 & 0 & 0 & 0 & 0 & 0 & 0 & 0 & 0 & 0 & 0 & 0 & 7 & 7 & 7 \\
\hline $\begin{array}{l}\text { Juul or Juul-compatible Classic } \\
\text { Menthol cap }\end{array}$ & 0 & 0 & 0 & 0 & 0 & 0 & 0 & 0 & 0 & 0 & 0 & 0 & 0 & 1 & 1 & 1 \\
\hline $\begin{array}{l}\text { Juul or Juul-compatible Crème } \\
\text { Brulee cap }\end{array}$ & 0 & 0 & 0 & 0 & 0 & 0 & 0 & 0 & 0 & 0 & 0 & 0 & 0 & 3 & 3 & 3 \\
\hline $\begin{array}{l}\text { Juul or Juul-compatible Fruit } \\
\text { Medley cap }\end{array}$ & 0 & 0 & 0 & 0 & 0 & 0 & 0 & 0 & 0 & 0 & 0 & 0 & 0 & 2 & 2 & 2 \\
\hline Juul-compatible yellow cap & 0 & 0 & 0 & 0 & 0 & 0 & 0 & 0 & 0 & 0 & 0 & 0 & 0 & 1 & 1 & 1 \\
\hline Juul Cool Mint 5\% 4-pack & 0 & 0 & 0 & 0 & 0 & 0 & 0 & 0 & 0 & 0 & 0 & 0 & 0 & 1 & 1 & 1 \\
\hline Juul Mango 5\% 4-pack & 0 & 0 & 0 & 0 & 0 & 0 & 0 & 1 & 0 & 1 & 0 & 0 & 0 & 0 & 0 & 1 \\
\hline Juul unknown 4-pack & 0 & 0 & 0 & 0 & 0 & 0 & 0 & 1 & 0 & 1 & 0 & 0 & 0 & 0 & 0 & 1 \\
\hline $\begin{array}{l}\text { Total no. of little cigar or cigarillo } \\
\text { items }\end{array}$ & 8 & 26 & 37 & 0 & 71 & 4 & 0 & 3 & 9 & 16 & 0 & 0 & 0 & 0 & 0 & 87 \\
\hline Little cigar or cigarillo wrappers & 7 & 17 & 26 & 0 & 50 & 3 & 0 & 0 & 7 & 10 & 0 & 0 & 0 & 0 & 0 & 60 \\
\hline $\begin{array}{l}\text { Little cigar or cigarillo mouth } \\
\text { pieces or butts }\end{array}$ & 1 & 9 & 11 & 0 & 21 & 1 & 0 & 3 & 2 & 6 & 0 & 0 & 0 & 0 & 0 & 27 \\
\hline Total no. of cigarette butts" & 8 & 42 & 193 & 32 & 275 & 59 & 33 & 6 & 15 & 113 & 12 & 27 & 103 & 90 & 232 & 620 \\
\hline Identifiable cigarette butts & 8 & 40 & 65 & 29 & 142 & 51 & 25 & 1 & 15 & 92 & 4 & 23 & 83 & 59 & 169 & 403 \\
\hline Marlboro menthols & 1 & 6 & 10 & 1 & 18 & 5 & 2 & 0 & 2 & 9 & 0 & 4 & 2 & 5 & 11 & 38 \\
\hline Newport menthols & 1 & 15 & 22 & 1 & 39 & 4 & 0 & 0 & 6 & 10 & 0 & 0 & 1 & 0 & 1 & 50 \\
\hline Camel menthols & 2 & 4 & 9 & 0 & 15 & 0 & 6 & 1 & 2 & 9 & 0 & 3 & 8 & 12 & 23 & 47 \\
\hline All other menthols & 0 & 6 & 7 & 0 & 13 & 2 & 3 & 0 & 2 & 7 & 0 & 0 & 11 & 2 & 13 & 33 \\
\hline $\begin{array}{l}\% \text { of menthol among all } \\
\text { identifiable butts }\end{array}$ & 50 & 78 & 74 & 7 & 60 & 22 & 44 & 100 & 80 & 38 & 0 & 30 & 27 & 32 & 28 & 45 \\
\hline Total no. of cannabis items & 2 & 3 & 0 & 0 & 5 & 4 & 0 & 0 & 3 & 7 & 0 & 0 & 0 & 2 & 2 & 14 \\
\hline Butts (roaches) & 1 & 0 & 0 & 0 & 1 & 1 & 0 & 0 & 0 & 1 & 0 & 0 & 0 & 0 & 0 & 2 \\
\hline Cartridges/Mouthpieces & 1 & 0 & 0 & 0 & 1 & 1 & 0 & 0 & 1 & 2 & 0 & 0 & 0 & 2 & 2 & 5 \\
\hline $\begin{array}{l}\text { High-potency oil concentrate } \\
\text { packaging }\end{array}$ & 0 & 3 & 0 & 0 & 3 & 2 & 0 & 0 & 2 & 4 & 0 & 0 & 0 & 0 & 0 & 7 \\
\hline
\end{tabular}

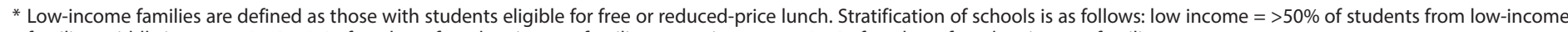
families; middle income $=25 \%-50 \%$ of students from low-income families; upper income $=<25 \%$ of students from low-income families.

+ National Center for Education Statistics, 2016. https://nces.ed.gov/ccd/schoolsearch/.

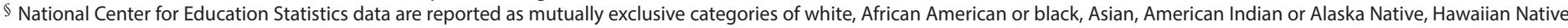
or other Pacific Islander, Hispanic or Latino, or multiracial.

Total cigarette butts do not equal sum of items because categories overlap. 
among U.S. youths. Further research and actions at national, state, and community levels are needed to inform policymaking to reduce youth access to and use of tobacco products, including e-cigarettes, and cannabis products. Youth use of flavored tobacco products, including mint and all other mentholated flavors, is of particular concern. Likewise, measures are needed to eliminate environmental contamination from e-cigarette, combustible tobacco product, and cannabis product waste in and around schools. Schools can engage students in garbology projects to identify existing and new use of these products and to raise awareness about their hazardous health and environmental impacts.

\section{Acknowledgments}

Gia Asher; Jasmine Gerraty, Kelsey Fernandez, Linda Henn, Iyo Fujita, Kazuyoshi Fujita, Masayuki Fujita, Marin Health Youth Partnerships, Larkspur, California; Office of National Drug Control Policy, Washington, DC; Substance Abuse and Mental Health Services Administration, Rockville, Maryland; California TobaccoRelated Disease Research Program, Oakland, California; National Cancer Institute, Bethesda, Maryland; Marin County Tobacco Control Program, San Rafael, California.

Corresponding author: Jeremiah Mock, jeremiah.mock@ucsf.edu.

\footnotetext{
${ }^{1}$ Institute for Health and Aging, Department of Social and Behavioral Sciences, University of California, San Francisco; ${ }^{2}$ Marin Healthy Youth Partnerships, Larkspur, California; ${ }^{3}$ Dynamics of Inclusive Prosperity Initiative, Erasmus School of Philosophy, Erasmus University Rotterdam, Netherlands; ${ }^{4}$ Environmental Health Initiative, University of California, San Francisco.
}

All authors have completed and submitted the International Committee of Medical Journal Editors form for disclosure of potential conflicts of interest. No potential conflicts of interest were disclosed.

\section{References}

1. Gentzke AS, Creamer M, Cullen KA, et al. Vital signs: tobacco product use among middle and high school students_-United States, 20112018. MMWR Morb Mortal Wkly Rep 2019;68:157-64. https://doi. org/10.15585/mmwr.mm6806e1

2. Food and Drug Administration. 2018 NYTS data: a startling rise in youth e-cigarette use. SilverSpring, MD:USDepartmentofHealthand Human Services, Food and Drug Administration; 2019. https://www.fda.gov/tobacco-products/ youth-and-tobacco/2018-nyts-data-startling-rise-youth-e-cigarette-use

3. Cullen KA, Liu ST, Bernat JK, et al. Flavored tobacco product use among middle and high school students-United States, 2014-2018. MMWR Morb Mortal Wkly Rep 2019;68:839-44. https://doi.org/10.15585/mmwr. mm6839a2

4. Kostygina G, Tran H, Shi Y, Kim Y, Emery S. 'Sweeter than a Swisher': amount and themes of little cigar and cigarillo content on Twitter. Tob Control 2016;25(Suppl 1):i75-82. https://doi.org/10.1136/ tobaccocontrol-2016-053094

5. Knapp AA, Lee DC, Borodovsky JT, Auty SG, Gabrielli J, Budney AJ. Emerging trends in cannabis administration among adolescent cannabis users. J Adolesc Health 2019;64:487-93. https://doi.org/10.1016/j. jadohealth.2018.07.012

6. Krause MJ, Townsend TG. Hazardous waste status of discarded electronic cigarettes. Waste Manag 2015;39:57-62. https://doi.org/10.1016/j. wasman.2015.02.005

7. Hendlin YH. Alert: public health implications of electronic cigarette waste. Am J Public Health 2018;108:1489-90. https://doi.org/10.2105/ AJPH.2018.304699

8. Novotny TE, Lum K, Smith E, Wang V, Barnes R. Cigarettes butts and the case for an environmental policy on hazardous cigarette waste. Int J Environ Res Public Health 2009;6:1691-705. https://doi.org/10.3390/ijerph6051691 